\title{
Three State-of-the-Art Methods for Condition Monitoring
}

\author{
Hugo T. Grimmelius, Peter P. Meiler, Hans L. M. M. Maas, Bas Bonnier, \\ Jasper S. Grevink, and Robert F. van Kuilenburg
}

\begin{abstract}
This paper describes and compares three different state-of-the-art condition monitoring techniques: first principles, feature extraction, and neural networks. The focus of the paper is on the application of the techniques, not on the underlying theory. Each technique is described briefly and is accompanied by a discussion on how it can be applied properly. The discussion is finished with an enumeration of the advantages and disadvantages of the technique. Two condition monitoring cases, taken from the marine engineering field, are explored: condition monitoring of a diesel engine, using only the torsional vibration of the crank shaft, and condition monitoring of a compression refrigeration plant, using many different sensors. Attention is also paid to the detection of sensor malfunction and to the user interface. The experience from the cases shows that all techniques are showing promising results and can be used to provide the operator with information about the monitored machinery on a higher level. The main problem remains the acquisition of the required knowledge, either from measured data or from analysis.
\end{abstract}

Index Terms - Fault diagnosis, feature extraction, modeling, monitoring, neural network applications, signal processing.

\section{INTRODUCTION}

C ONDITION monitoring has always been applied. In the early days, monitoring was performed by the people who actually operated the machinery. Because they worked physically close to the machinery they were operating, they could assess its condition directly, based on experience. When the machinery became more complex, measurement systems were installed to make information about its condition available to the operators at a central operating console, in a ship, for instance, the engine control room and/or the bridge (Fig. 1). The number of operators decreases, but they still have to be kept informed about the state of the machinery. To prevent information overload, the condition monitoring process must be automated to provide the information on a higher level and at the desired location. Automation also makes condition mon-

Manuscript received February 26, 1997; revised July 1, 1998. Abstract published on the Internet January 18, 1999. This work was supported in part by The Dutch Institute for Maritime Research (CMO/NIM)

H. Grimmelius is with Delft University of Technology, 2628 CD Delft, The Netherlands.

P. P. Meiler is with the Platform Command and Control Group, TNO Physics and Electronics Laboratory, 2509 JG, The Hague, The Netherlands.

H. L. M. M. Maas is with the Combat Direction Systems Section, TNO Physics and Electronics Laboratory, 2509 JG, The Hague, The Netherlands.

B. Bonnier is with MHI Equipment Europe, 1303 AC, Almere, The Netherlands.

J. S. Grevink is with Lips Propeller Works, 5150 BB, Drunen, The Netherlands.

R. F. van Kuilenburg is with INNAS B.V., 4823 AE, Breda, The Netherlands.

Publisher Item Identifier S 0278-0046(99)02720-3. itoring more accurate and provides faster response times. This allows a more critical design of machinery and smaller design margins, and will result in a reduction of the life-cycle costs. Modern condition monitoring systems may also be augmented by a system that advises the personnel on actions to be taken when certain faults occur. As a result, operating costs and reliability can be improved significantly. As shown in Fig. 2, condition monitoring systems measure process variables on the machinery. Using one or more sensors, these measurements can be done continuously or at specific intervals. Modern sensors provide the ability to monitor machinery at locations that are difficult to access (like the pressure in a cylinder). First, the sensor signals are preprocessed. At this stage, sensor failure(s) should be detected, and the correct signals are transformed to a suitable format for the processing stage. In the processing stage, the condition of the machinery is determined. This can be implemented using several methods, three of which are described in this paper. Finally, the results of the earlier stages are postprocessed for a clear presentation to the user. In the first part of this paper, the preprocessing and the signal processing methods are described. Each of the signal processing methods is described, as much as possible, independently of the application. Special attention is given to the required process knowledge and sensor information for each method, as well as the advantages and disadvantages of each method. Consideration is also given to sensor fault detection and to the user interface. In the latter part, the three processing methods are "tested" on two real-world applications, a ship's diesel engine and a refrigeration plant.

\section{SENSORS AND PREPROCESSING}

Condition monitoring systems depend on sensors for obtaining the necessary information. However, the odds of sensor failure are often of the same order of magnitude as the odds of machinery failure. Since the diagnosis determined by a condition monitoring system can only be accurate if the measured information is correct, the first step should be to evaluate the sensor signals. The reliability of the sensor system can be increased by monitoring the sensors themselves. Sensor fault detection methods can be divided into direct and modelbased methods. Direct methods are based on an evaluation of the actual sensor signals. Some examples follow.

Sensor Redundancy: Double redundant sensors can indicate the failure of one of the sensors, but cannot tell which. Triple redundant sensors can (in most cases) locate the failing sensor. 


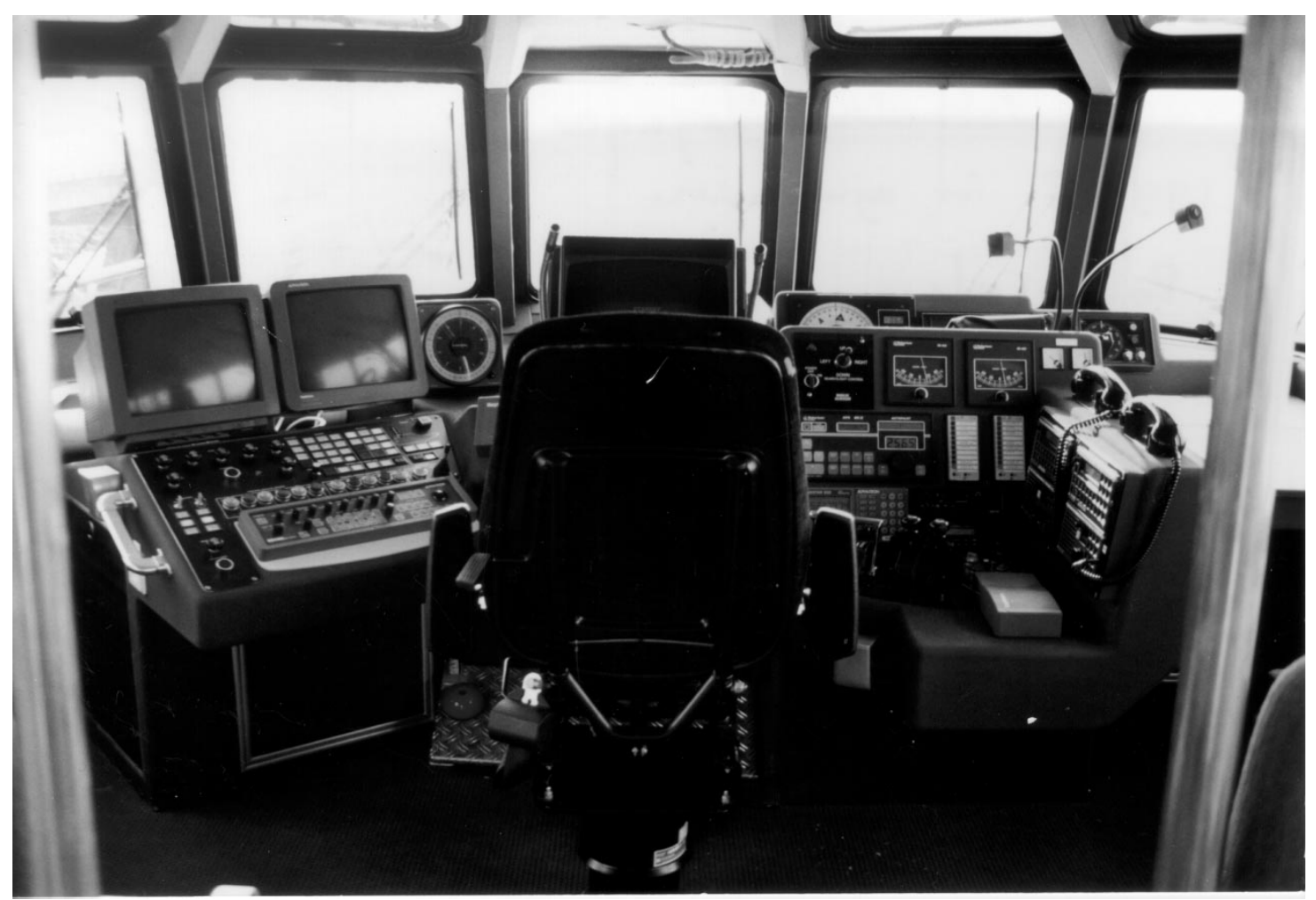

Fig. 1. Bridge of a modern ship.

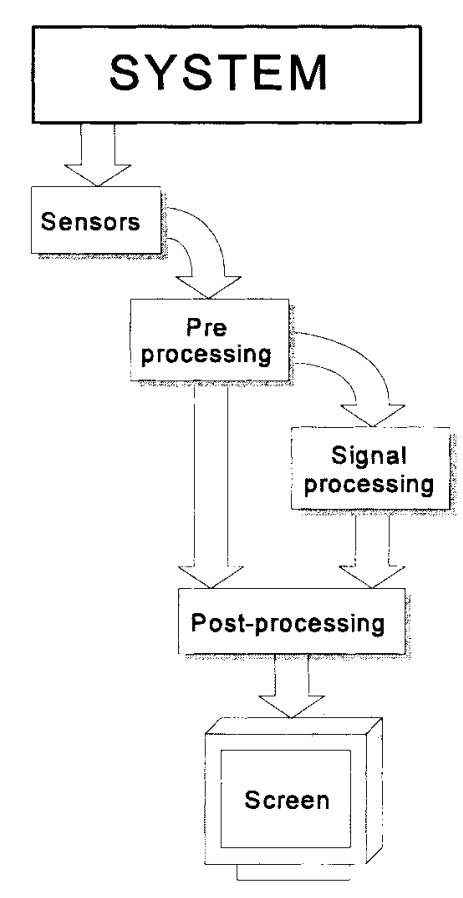

Fig. 2. Condition monitoring.

Range Checking: The use of sensors with a limited range enables range checking.

Off-Period Values: After the machinery is stopped, the temperatures and pressures will stabilize at specific off-period values. Deviations in the off-period sensor values can point to sensor failures.
Model-based methods use information about the monitored machinery to create an "analytic" sensor redundancy. Instead of using two (or more) redundant sensors, the model will function as one of the redundant sensors. These methods can identify less prominent sensor faults than the direct methods mentioned above. Two successful methods are mentioned here.

Observer-Based Sensor Monitoring: Based on models of parts of the machinery and other sensor values, several observers calculate an estimate for the value of a specific sensor. These estimated values are redundant with the measured values and, thus, give an indication of a sensor fault.

Sensor Fault Analysis [3]: If a sensor fails, a characteristic pattern will appear in the measured sensor data. This pattern is unique for a specific sensor fault.

\section{Signal Processing Methods}

\section{A. Introduction}

Storing and displaying sensor values only shifts the point of view from, for instance, the engine room to the control room or the bridge, without giving the operator support for the interpretation of these values. This requires process knowledge, that is, knowledge about the machinery and its behavior. The methods presented here use different amounts of process knowledge, as illustrated in Fig. 3.

The amount of available process knowledge limits the applicable methods. The choice of a method, however, is determined by the required performance and may not require all available process knowledge. Machinery diagnosis implies detection of, and distinguishing between, faults by processing 


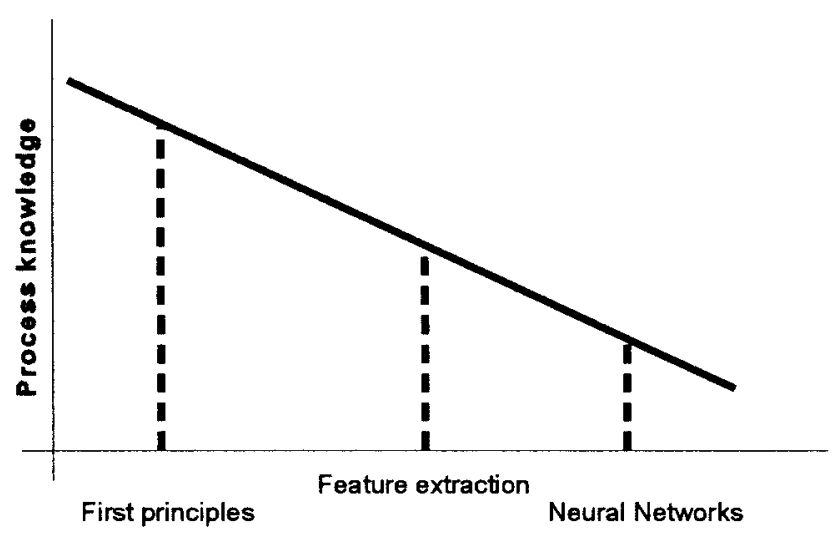

Fig. 3. Required process knowledge.

measured symptoms or features. The following three basic methods are presented in this paper:

1) signal processing using models based on first principles;

2) signal processing using feature extraction and pattern recognition;

3) signal processing using neural networks.

\section{B. First Principles Method}

The first principles method uses mathematical simulation models based on process physics to predict the behavior of machinery, both for healthy and faulty conditions. One of the main characteristics of these simulation models is the required high level of knowledge of the machinery and the processes. They require extensive knowledge of the "first principles," such as conservation laws, and of constitutional laws, such as the properties of matter. Some measured data is required for tuning, validation, and verification.

There are two principally different ways to utilize first principles models within the field of condition monitoring.

1) The models can be used to analyze the actual measured behavior of the machinery. On-line application is, for instance, establishing the healthy behavior of the machinery, given the operational parameters. This is an effective approach for finding a fault before it can cause more damage to the machinery. Problems may occur when two different faults have the same influence on the measured signal. This occurs if the model is not reversible. By using a model, insight is gained into the behavior of the machinery. This insight is not only useful for condition monitoring, but can also be used for machinery improvement, training purposes, or development.

2) The models can be used to gain knowledge about the behavior of machinery when a fault occurs. The structure of the models has to be such that faults can be introduced unambiguously. Because faults cause off-design behavior, existing models can usually not be used. Depending on the level of detail of the models, only a limited number of faults is available for simulation. Because faults create off-design conditions, no data-measured or supplied by the manufacturer-are available to vali- date the results. This puts high demands on the reliability of the modeling concept. It is expected that only the qualitative results of the fault simulations will be reliable, since validation of the models is not possible. For machinery which consist of complex components, the development of new models is very costly. For complex machinery which consist of relatively simple components, the development of a library of standard component models could be considered (also due to the high costs that are involved in developing models). The application of this method would then require far less effort. This approach could well be suited for machinery consisting of a large number of components, such as pneumatic, hydraulic, and electronic systems.

The advantages of this method are as follows.

1) The user of the condition monitoring system does not need the process knowledge to interpret the measured signal.

2) Much insight into the behavior of the machinery is gained, and this insight can be used for other applications or for further development of the monitored machinery.

3) Only a limited amount of measured data is needed.

4) The knowledge necessary to diagnose faults correctly is gained in a structured way and is reproducible.

5) The diagnostic knowledge required for the condition monitoring system is available on startup.

6) The method is well suited for newly designed, complex machinery.

7) The availability and reliability of the knowledge is no longer dependent on the experience and subjective judgement of human experts.

The disadvantages are as follows.

1) A high accuracy of the measured signals is required.

2) The model is always an approximation of the real machinery.

3) Thorough process knowledge is needed for development of the model.

4) A new model has to be designed for every type of machinery.

5) The knowledge gained is mainly qualitative; quantitative results are often unreliable.

6) Only a limited subset of faults is simulated.

\section{Feature Extraction and Pattern Recognition Method}

Feature extraction and pattern recognition algorithms are used for analyzing signals and for classifying (parts of the) signals into classes. The classification is done by matching (part of) the signal with a set of reference signals. The sensor signal will be classified as a member of the class that corresponds with the best matching reference signal.

The isolation of parts of the signal that are unique for the classes results in a better control of the classification problem. In this way, the influence of fluctuations in the sensor signal which are caused by instabilities and noise will be reduced to a minimum. The process of isolating those parts of the sensor signal is called the feature extraction process, while the matching process is known as pattern recognition. The 
isolation of features from the sensor signal has the benefit that several faults can be detected independently, using the same signal, if there is no correlation between the patterns of the features for the different faults. Process knowledge is only needed to indicate the (expected) behavior of the sensor signals. This implies that this method is suitable to monitor machinery for which an accurate enough model is not available. This method requires a mathematical description, or at least a lookup table for each feature that corresponds with a fault, over a wide range of operating conditions. As a result, a lot of effort is needed to develop the feature extraction and classification algorithms.

Feature extraction and pattern recognition can be extended with new functions for the detection of new faults without changing already existing fault detection functions. A modular construction has the benefit that already existing modules can be reused in the implementation of new functions.

The advantages of this method are as follows.

1) Little process knowledge is needed.

2) The algorithms are only triggered by predefined patterns of the sensor signals. This reduces the influence of noise.

3) The decision process is traceable. The usage of welldefined algorithms makes it possible to tell which classification corresponds with which sensor and provides insight in the underlying reasoning process.

4) The method is suitable for machinery for which a mathematical model is not available.

The disadvantages are as follows.

1) Measurements of faults are required.

2) Detailed knowledge of the behavior of the sensor signal(s) is needed to determine which parts of the signals are relevant.

3) Much effort is needed to develop the algorithms.

4) Depending on the complexity of the signals, highperformance computing techniques might be needed.

\section{Neural Network Method}

Neural network technology is used to recognize and classify complex fault patterns without much knowledge about the process, the signals, or the fault patterns themselves. A neural network consists of many simple neurons which are connected with each other (Fig. 4). The behavior of the network is determined by the (adjustable) weights that are associated with each connection. The values of these weights are determined during the training session. During this session, examples of the different situations (input patterns with corresponding output classifications) are presented to the neural network. Neural networks tend to be very robust to noise in the signals [2]. Application of neural network technology requires a large training data set, covering all classes of conditions that are required to be detected. The results of a neural network are only valid within the range of this training data set. On the other hand, the ability of neural networks to "train" themselves sometimes allows neural networks to find a solution where other methods fail. Choosing the correct network layout and training methods for a certain task is difficult. There is little structured knowledge about which network and training

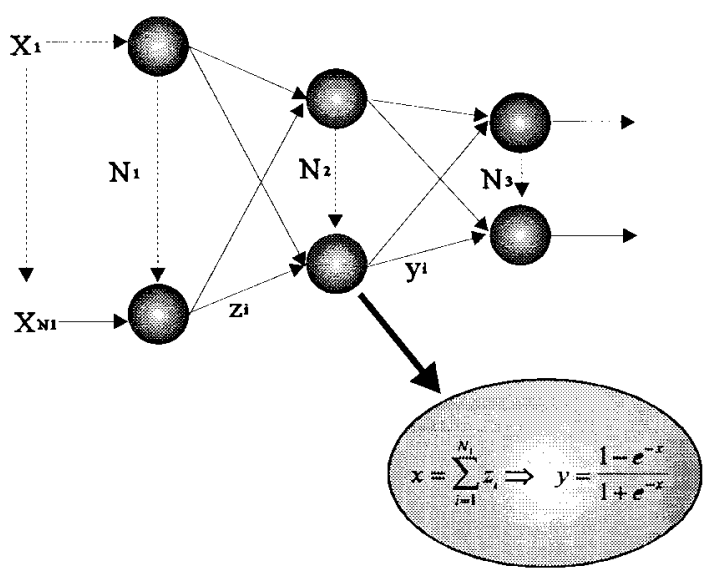

Fig. 4. A neural network.

method are best for a certain application. However, this choice is crucial for obtaining a good result. Neural-network-based systems do not provide insight into the classification criteria that the network is trained to use. It is never possible to prove the quality of such a system deterministically. Using statistical techniques (based on a separate test data set), the performance of such a system can be estimated.

The advantages of this method are as follows.

1) Neural networks are very fast. This is useful when a diagnosis system must run in real time and must handle a lot of signals.

2) Little or no process knowledge is required using neural networks. A special advantage is that no knowledge about fault patterns is required.

3) A neural network is robust, especially regarding noise.

The disadvantages are the following.

1) Knowledge about, and a "feel" for, the training of neural networks is required.

2) Training neural networks is time consuming.

3) An extensive set of measured data is required for all classes of conditions, including faults.

4) The required data sets can be very large.

5) A neural network does not provide insight into the criteria it uses to classify the input patterns, because the learned knowledge is distributed over all the weights in the network.

6) A neural network only provides valid answers inside the trained range.

7) A neural network, once trained, is not flexible. If environmental conditions change to values outside the trained range, the network must be retrained.

\section{PostProcessing AND the USER INTERFACE}

After the sensor preprocessing and fault detection have been done, using one of the previously discussed methods, this information should be presented to the operator. The use of screens can improve the presentation of information significantly. Not only the regular process variables and the condition monitoring diagnosis can be displayed, but also maintenance advise can be given and trend analysis can 


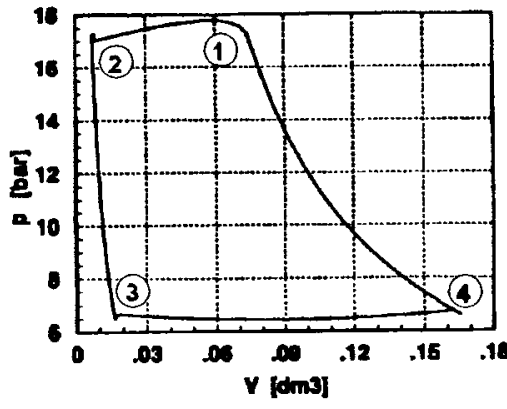

(a)

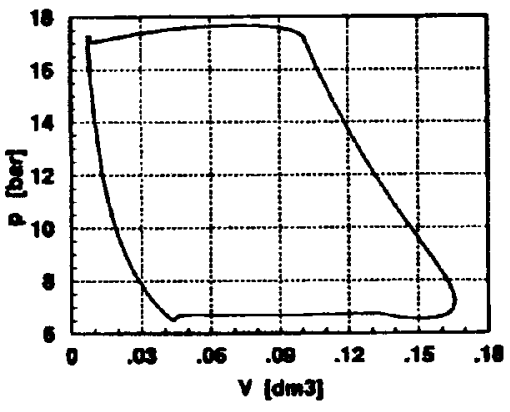

(b)

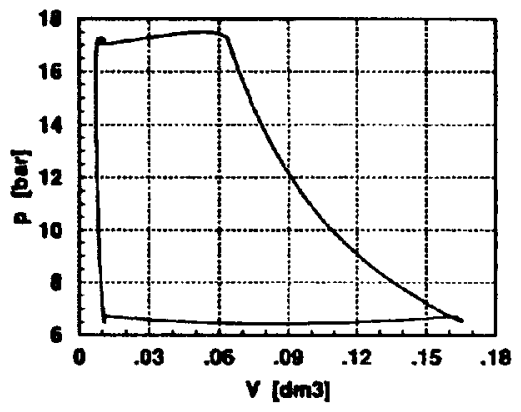

(c)

Fig. 5. p-V diagram. (a) Healthy. (b) Leaking discharge valve. (c) Leaking suction valve.

be performed. The design of the user interface should be ergonomic, while the layout and the use of the user interface must be simple and comprehensible. For condition monitoring systems, it is useful to distinguish between two types of displays, survey displays and specific displays [6]. Both can be either static or dynamic. During regular operation, the survey displays present the status of the machinery, using key information and graphic tools. Only when the diagnosis module reports problems, the operator must look "deeper" into the specific displays of the user interface where the problem is identified.

The information which should be presented by the user interface is the following:

1) values of process variables;

2) status of the machinery through processed information (e.g., percentage load, heat flows, machinery and sensor faults);

3) past, present, and predicted values of condition parameters (advises for problem solving);

4) whether or not the machinery is in a stationary condition.

\section{CASES}

To illustrate the use of the signal processing methods, two cases are described. The cases are taken from the marine engineering field.

\section{A. The Refrigeration Plant Case}

Compression refrigeration plants are widely used on board ships for cooling of perishable cargo and machinery, for air conditioning and for victuals. Frequently, faults in the plant are not recognized before a breakdown occurs, because symptoms that could be used to diagnose a defect are difficult to discern from noise. Furthermore, given a set of deviations, even experts are often not sure about the cause. This lack of condition monitoring and diagnostic knowledge is the result of the closed-cycle character of the process and of the strong dependency of measurable variables on the operating conditions. The lack of knowledge is amplified by the high reliability and maintenance-free operation of these plants, reducing the "hands on" experience of the operators. In [4], a prototype condition monitoring system was developed to evaluate the possibilities and problems of such a system. The two main problems are: 1) predicting the behavior of the plant when a fault occurs and 2) reliable recognition of the symptoms caused by a defect, especially when only a few symptoms are to be expected. In this case, the first problem is addressed.

1) Refrigeration Plant Case-The Use of First Principles Models: Prediction of the behavior of the plant, based on a general process description, often proves to be insufficient. The behavior depends too strongly on the operating conditions and on the specific dimensions and layout of the plant to allow a detailed diagnosis. Therefore, a detailed model of the plant is being developed which enables the simulation of faults [5]. The basis for this model is a description of the refrigerant flow in terms of resistors (valves) and storage elements (vessels). Added to this description are the energy flows in the various components. This results in a model that is capable of simulating the dynamic behavior of the plant at various operating conditions, with and without the presence of a fault.

a) Examples: The reciprocating compressor model renders a good match, both with data provided by the manufacturer and with actual measurements. Fig. 5(a) shows a typical pressure versus volume $(\mathrm{p}-\mathrm{V})$ diagram produced with the compressor model at a condenser pressure of $17.3 \mathrm{bar}$ and an evaporator pressure of 6.8 bar. The results of two fault simulations are presented to show the possibilities of such a detailed model. Fig. 5(b) shows the p-V diagram for the same condition, but with a leaking discharge valve, calculated with the model. It shows that the leaking valve has a large influence on the compression and expansion curve. The expansion is lengthened by the refrigerant flowing back through the discharge valve, whereas the compression is shortened. The work performed per cycle increases, while the mass flow decreases. In Fig. 5(c), the p-V diagram is plotted for a simulation with a leaking suction valve. During the expansion, gas is leaking out of the cylinder and, contrary to Fig. 5(b), the expansion has become shorter. The compression has become longer for the same reason. The work performed per cycle and the mass flow both decrease. These examples show that straightforward faults, such as leaking valves may cause different plant behavior, depending on the location of the fault (suction or discharge), especially when the faults are considered at an early stage of their development. It has 


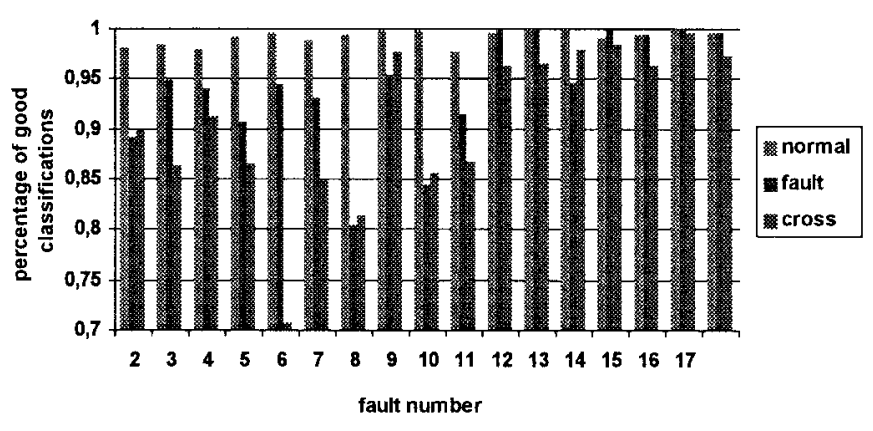

Fig. 6. Result of neural networks.

proven to be difficult to create models that are both flexible and reasonably detailed. The results are promising, both for simulation of healthy behavior and for simulation of faults.

2) Refrigeration Plant Case-The Use of Neural Networks: If the symptoms caused by a fault are known, for instance, through the use of a detailed model or through measurements, it still is often difficult to discern them in the measured signals. In this case, neural networks were used to generate a diagnosis about the health of a refrigeration plant, based on data from extensive measurements, including seventeen faults [7]. To enhance the flexibility and to be able to add more faults in the future, a separate network was trained to recognize each fault. Each network is trained with a different training data set. All networks have 11 input neurons and two output neurons. The number of hidden neurons varies, depending on the fault to be recognized. The results of the different networks are shown in Fig. 6. This figure shows that the results that can be reached by this kind of method are very good. First, if the refrigeration plant runs normally, the neural networks recognize this with great accuracy, more than $95 \%$ of all data points are diagnosed correctly as "normal." Secondly, when a fault has been introduced into the plant, the corresponding network also recognizes this fault with almost the same accuracy. The lower score is mostly due to the fact that the data files which contain the data of the faults, also contain some healthy system data. Finally, the correct fault has to be recognized. Fig. 6 shows that the results for this "cross" check are also very good. It is stressed that all the faults can be recognized. The performance of the neural networks is calculated without postprocessing. When using an appropriate postprocessing method, such as a majority vote, the results can be even better. Another point to be taken into account is that it is important to recognize a fault before serious damage to the machinery has occurred. This implies that misdiagnosing a few data points (false alarms) at an early stage need not be a problem, provided that the fault is detected on time.

\section{B. The Diesel Engine Case}

The purpose of this feasibility study was to detect faults in the combustion of one of the five cylinders of a medium-size diesel engine from the torsion signal at the crankshaft of the engine. All three methods have been evaluated by applying it to the test engine. Data was measured and processed for healthy conditions and for conditions with artificially induced

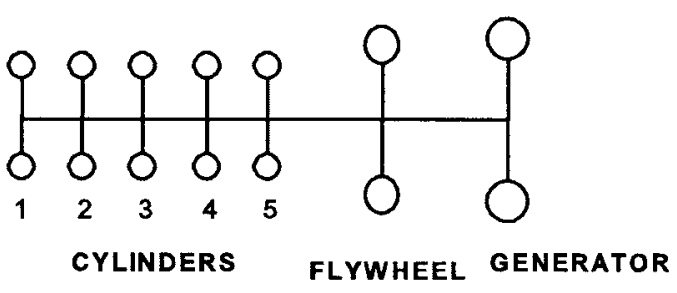

Fig. 7. Lumped mass model.

defects on one or more cylinders, such as nonfiring, partial fuelling, leakage, and a faulty fuel injector.

1) Diesel Engine Case-The Use of First Principles Models: To monitor the condition of a diesel engine, a model is made of the rotor system of the engine. The engine dynamics are described by a lumped mass model, shown in Fig. 7. This model has been used to calculate the vibrations of the rotor system during one firing sequence. If the gas pressures are known, the vibrations of the engine can be calculated with this model, resulting in Fig. 8. The gas pressures can be actually measured, or can be determined using the "Tangential effort curves" published by Lloyd's Register. In principle, the rotor system model can also be used for reverse calculation of the cylinder gas pressures using measured vibrations. In practice, however, application of this method is restrained by the limited amount of information available for this purpose in the response. Additional information can be obtained from the distinct shape of the cylinder gas pressure at a varying load. Gas pressures are split into two parts, one part caused by compression and expansion and the other part caused by combustion, as shown in Fig. 9. The combustion part is scaleable, which makes it possible to define an invariable standard curve for the pressure rise due to combustion. This is multiplied by a factor to account for the variable cylinder load. Thus, a simple gas model with only one variable-the load-for each cylinder is obtained. The limited amount of information in the response is sufficient for a reverse calculation of all cylinder pressures using a best fit procedure for the measured and calculated vibrations [10]. It was possible to calculate the individual cylinder power with an accuracy of $10 \%$ using one torsional vibration signal. Fig. 10 shows the power participation factors of all individual cylinders, both measured and calculated. Faults can be located easily in an early stage so damage to the machinery can be limited.

2) Diesel Engine Case-The Use of Feature Extraction and Pattern Recognition: The purpose of this part of the research was to find features which could be used to detect a cylinder firing failure in one or more of the five cylinders using only the torsion signal. Fig. 11 shows the torsional vibration signal for three different conditions of the diesel engine during one firing cycle (two revolutions). Fig. 11(a) represents the torsion signal of a healthy diesel engine, while Fig. 11(b) and (c) show the torsion signals of a diesel engine when cylinder 1 run at partial power and when cylinder 1 is turned off, respectively. The figure shows that an imbalance of the diesel engine influences at least the zeroth and first harmonics of the torsion signal. The figure also shows that the maximum torsion value of cylinder 1 , when it has a defect, has a higher value than the maximum 


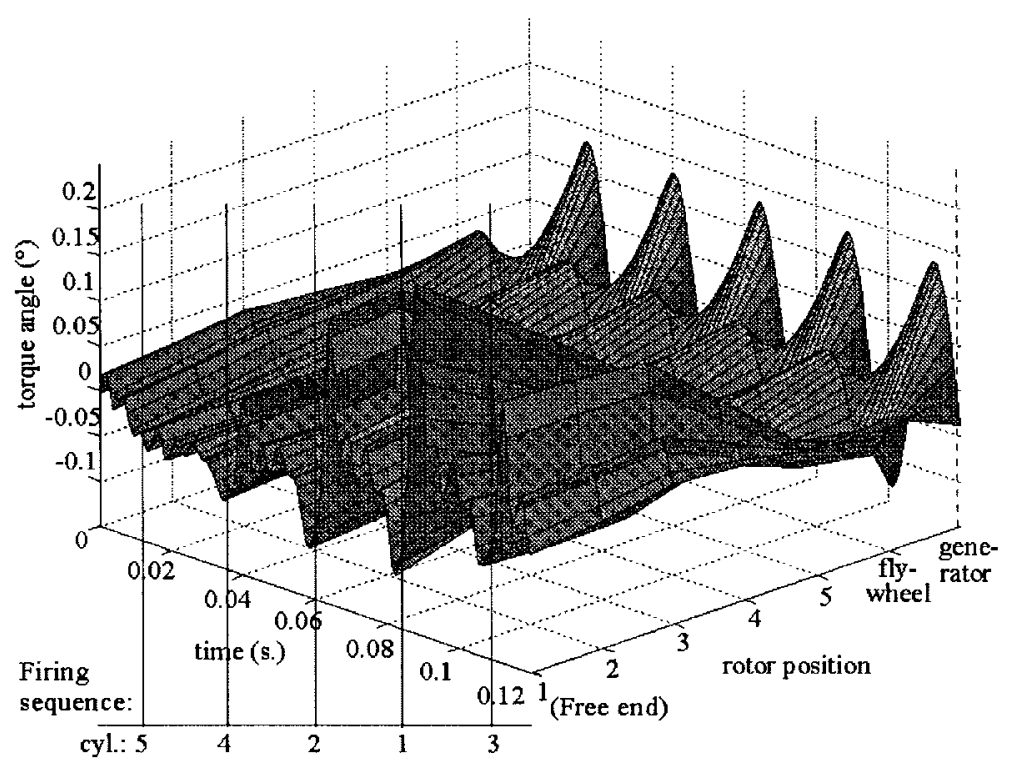

Fig. 8. Calculated vibrations.

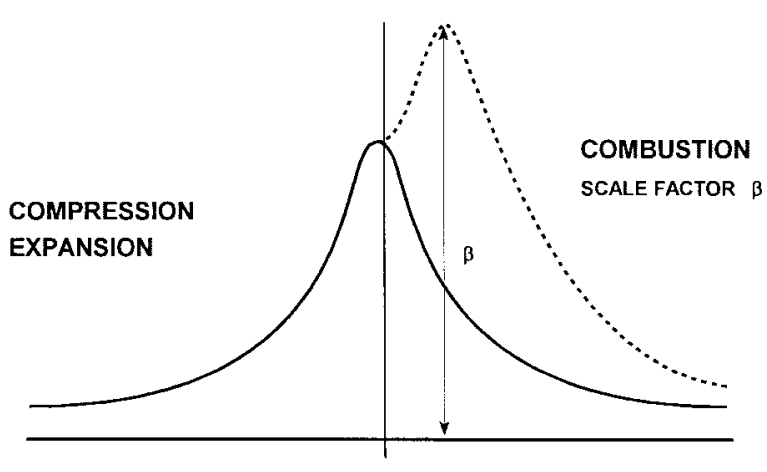

Fig. 9. Split of gas pressure.

\section{Cylinder 5: partially fueled}

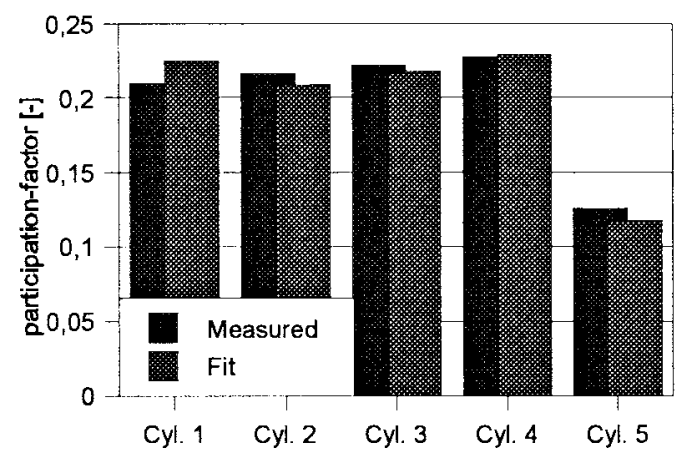

Fig. 10. Results of first principles diagnosis.

torsion value when cylinder 1 is healthy. These features are accentuated and isolated with the aid of two different analysis methods [9], base-level fluctuation (BLF) signal analysis and torsion-peak value analysis.

a) BLF signal analysis: The BLF signal is derived from data measured at five fixed sample points in the torsion signal. These five points represent the location of the start of the compression of each cylinder. A cubic interpolation algorithm is used to determine the BLF signal function, as shown in Fig. 12. The amplitude of the interpolated signal gives an indication about the kind of defect. A large amplitude represents a cylinder-off failure, while a small amplitude represents a partial power failure. The crank angle at which the BLF function reaches its maximum value indicates which cylinder does not work well.

b) Torsion-peak analysis: The torsion-peak analysis first takes the difference between the torsion signal and the BLF signal. In the resulting signal, the defect is more visible than in the original signal. Fig. 13(b) shows that the peak value of cylinder 1 decreases if the cylinder is running at partial power. The peak value becomes even smaller after turning off cylinder 1, as shown in Fig. 13(c). The peak values of each cylinder are compared with the mean peak values of each cylinder for a healthy diesel engine. The ratios between the measured and the reference peak values give an indication about the condition of each cylinder of the diesel engine, and are classified according to: 1, the cylinder is classified as healthy; $<1$, the cylinder is working at partial power; and $\ll 1$, the cylinder is not working (power off). In contrast with the base-level function method, it is possible to monitor the condition for each cylinder separately.

The results of both the BLF signal analyses and the results of the torsion-peak analyses are used to compose one overall conclusion about the condition of the diesel engine.

3) Diesel Engine Case-The Use of Neural Networks: The torsion signal is also used directly as an input pattern to the neural network. The data set contains data of the healthy condition of the engine and data for the conditions where one or more defects are introduced or simulated. The backpropagation algorithm [2] was used to train several twolayer neural networks. First, a simple network was trained 


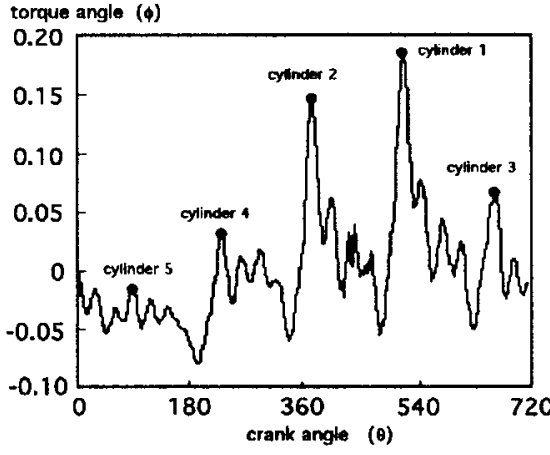

(a)

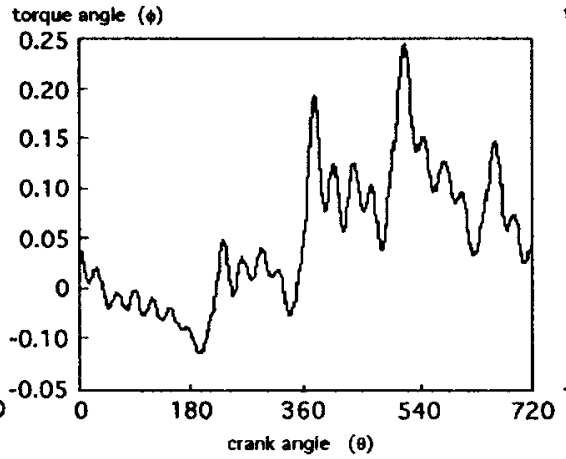

(b)

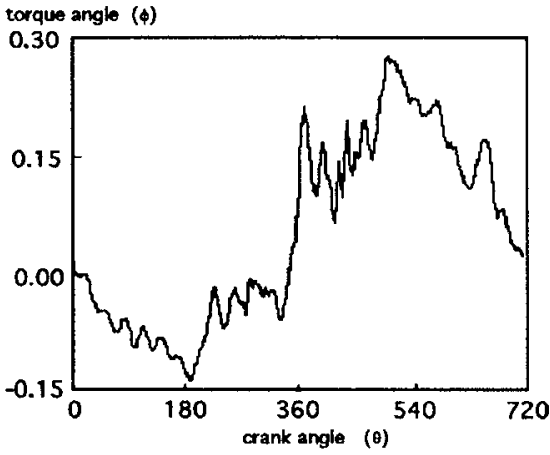

(c)

Fig. 11. Torsion signal for (a) healthy engine, (b) cylinder 1 running at partial power, and (c) cylinder 1 off.

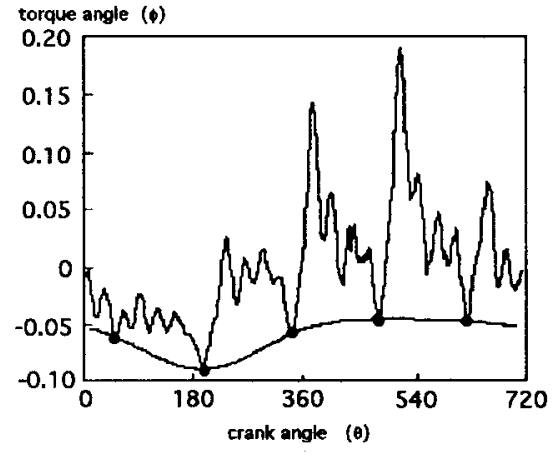

(a)

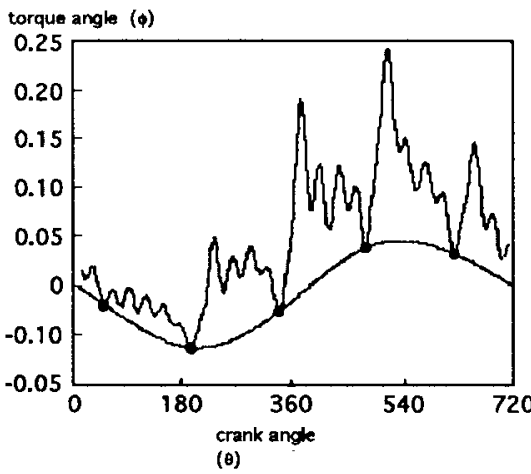

(b)

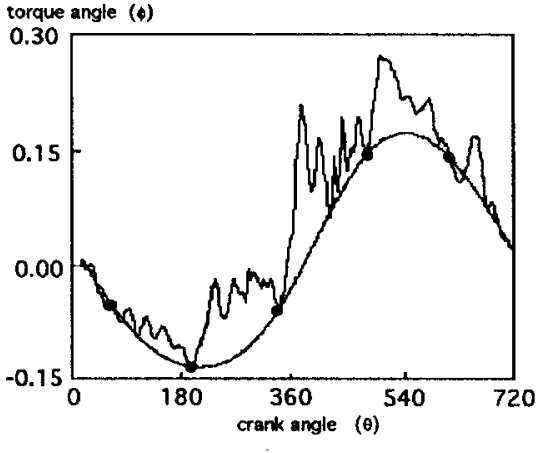

(c)

Fig. 12. BLF for (a) healthy engine, (b) cylinder 1 running at partial power, and (c) cylinder 1 off.

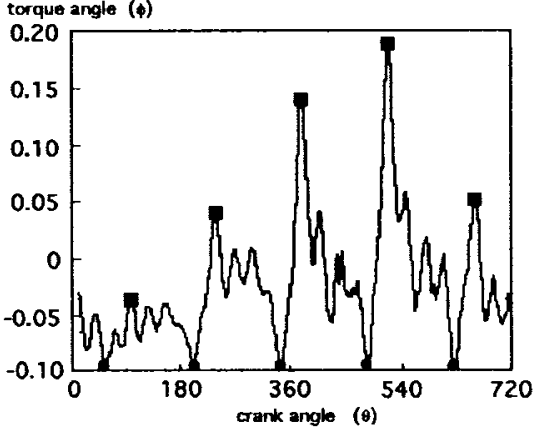

(a)

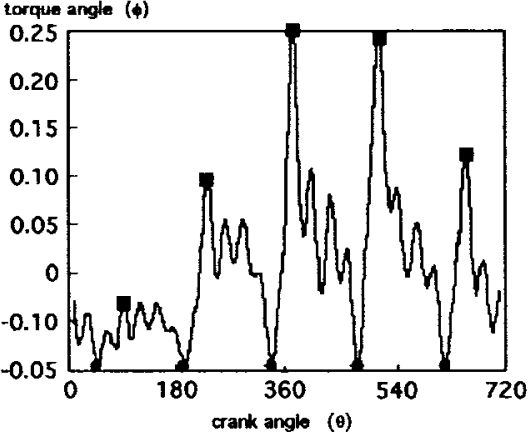

(b)

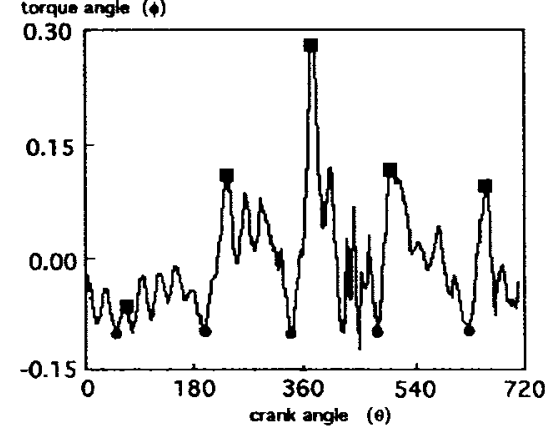

(c)

Fig. 13. Torsion-peak value for (a) healthy engine, (b) cylinder 1 running at partial power, (c) cylinder 1 off.

that could only discern between the healthy and not-healthy states. Progressively more complicated networks were trained, capable of classifying a growing combination of defects (e.g., healthy, cylinder-1 defect, cylinder-2 partial power, etc.). See also [9].

The training set was limited by the measurements that were available. The available data set consisted of 8 Gbytes. The measurement signals were preprocessed extensively using the following steps: resampling (to :synchronize" the signals in bins of 512 samples per combustion cycle), filtering, averag- ing, peak removal, and normalizing. Each of the 512 samples is mapped on one neuron of the input layer of the network. Each neuron in the output layer represents one class of defects. Postprocessing is done by only accepting a neural network classification if the neural network output values comply with certain threshold values and if the "distance" between the highest output neuron value and the second-highest output neuron value exceeds a threshold. Furthermore, classification will only be accepted if it has occurred for a minimum number of times within a fixed time period. Using the automated 
TABLE I

COMPARISON OF METHODS

\begin{tabular}{l|c|c|c}
\hline & Model based & Feature extraction & Neural networks \\
\hline 1. Required process knowledge & extensive & limited & minimal \\
\hline 2. Required initial data & minimal & limited & extensive \\
\hline 3. Development effort & extensive & extensive & limited \\
\hline 4. Computer power during development & limited & limited & extensive \\
\hline 5. Computer power on-line & extensive & limited & minimal \\
\hline 6. Maintainability & good & moderate & bad \\
\hline 7. Adaptability & good & moderate & bad \\
\hline 8. Acquired in-sight & extensive & limited & none \\
\hline
\end{tabular}

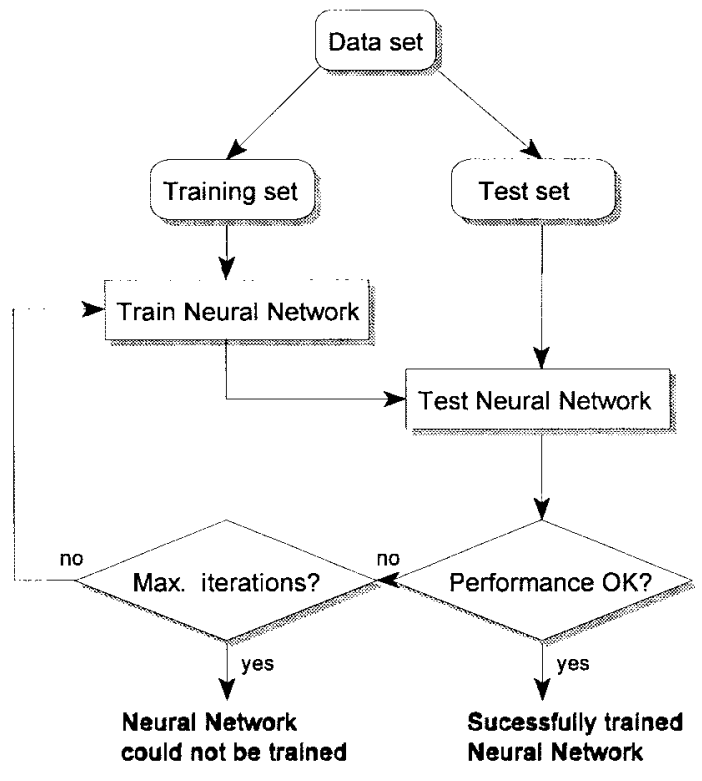

Fig. 14. Training of neural networks.

procedure shown in Fig. 14, the neural networks were trained to detect the following (combinations of) defects. The success rate is given as a percentage in the following:

1) detect which cylinder is off $(100 \%)$;

2) detect which cylinder is off or partially off $(78 \%)$;

3 ) detect which cylinder is off or partially off, for two independent faults, for cylinders 1, 2, and $3(100 \%)$;

4) detect late ignition $(75 \%)$;

5) detect early ignition (100\%);

6) detect low injection pressure $(83 \%)$;

7) detect leak in cylinder (100\%).

\section{EVALUATION}

Adding "intelligence" to the monitoring system can reduce the number of operating personnel and can minimize the damage by detecting changes in the condition of the machinery at an early stage. A main area for further development of condition monitoring systems is signal processing. This research shows that the three methods described are suitable for condition monitoring. A comparison on main characteristics for these methods is given in the matrix in Table I.

As discussed, the required process knowledge is an important issue in the development of a condition monitoring system. The required data is the data necessary to develop and test the models, to find the features, or to train the neural network. An important problem in this respect is the availability of data of the machinery for conditions where a fault is occurring. The development effort needed to build the signal processing module will also be dependent on the experience and expertise of the developers. The computing power needed during development is not the same as the computing power needed on-line. During development, available computing power can influence the required development time considerably. On-line, it influences the delay between measurement and result on screen.

Maintainability of the software indicates the possibilities to maintain and change the monitoring system after it has been implemented. Changing operating conditions require adaptability of the signal processing module. The insight acquired during the development of the signal processing module can be used for other purposes, like machinery development and improvement.

Model-based methods can be applied efficiently for newly developed machinery because the design data is already available. Feature extraction and neural networks strongly depend on the availability of measured data and are, therefore, more suited for application to existing machinery. The matrix shows that no single method is satisfactory in every respect. Therefore, a combination of the methods is likely provide the best results.

\section{ACKNOWLEDGMENT}

\section{ORGANIZATION OF THE RESEARCH AND PARTNERS INVOLVED}

The research described within this paper was done mostly within the scope of the Intelligent Condition Monitoring Systems (ICMOS) and Modern Information Technology Applied to Condition Monitoring (MIT) projects. The ICMOS project involved the Department of Mechanical Engineering and Marine Technology, Delft University of Technology (DUT), Stork Wärtsilä Diesel, Netherlands Shipbuilding 
Bureau (NEVESBU), The Royal Netherlands Navy (RNIN), and Van Buuren-Van Swaay Mechanical Contractors. Both the RNIN and Van Buuren-Van Swaay provided the opportunity to perform measurements on operational machinery. The MIT project, initiated by the RNIN, involved three partners, two within the Netherlands Organization for Applied Scientific Research (TNO), namely, the Physics and Electronics Laboratory (TNO-FEL) and the Centre for Mechanics and Information Processing Technology (TNO-MIT), and one within the Royal Netherlands Navy, namely, the Royal Netherlands Naval College (RNINC), which provided measurement data of, and process knowledge about, the diesel engine.

\section{REFERENCES}

[1] J. S. Bonnier, "Torsietrillingsanalyze ten behoeve van de conditiebewaking van zuigerverbrandingsmotoren," Delft Univ. Technol., Delft, The Netherlands, Rep. OEMO 94/08, pts. 1-3, Aug. 1995.

[2] W. S. Sarle. (Dec. 1998). Neural networks: FAQ, papers and various other things [Online]. Available FTP: //ftp.sas.com/pub/neural/FAQ.html

[3] J. S. Grevink, "Ontwikkeling van een conditiebewakingsmethodiek voor een dieselkoelwatersysteem," Delft Univ. Technol., Delft, The Netherlands, Rep. OEMO 96/05, May 1996.

[4] H. T. Grimmelius, J. K. Woud, and G. Been, "On-line failure diagnosis for compression refrigeration plants," Int. J. Refrigeration, vol. 18, no. 1, pp. 31-41, 1995.

[5] H. T. Grimmelius, "Modeling of a compression refrigeration plant for fault simulation," in Proc. 19th Int. Congr. Refrigeration, 1995, vol. IIIa, pp. 299-306.

[6] W. A. M. Hoefnagels, Ontwerpergonomie. Delft, The Netherlands: Delft Univ. of Technol., Mar. 1996, ch. 20.

[7] R. F. van Kuilenburg, "Fault diagnosis using neural technology," Delft Univ. Technol., Delft, The Netherlands, Rep. OEMO 96/01, May 1996.

[8] P. P. Meiler, H. L. M. M. Maas, H. S. T. Brockhoff, C. A. J. Tromp, and T. Popma, "Final report of the project "Modern Information Processing Technology (MIT)," TNO-FEL, The Hague, The Netherlands, FEL-97A255, Dec. 1998

[9] P. P. Meiler and H. L. M. M. Maas, "Condition monitoring of a diesel engine by analysing its torsional vibration, using modern information processing technology," in Proc. 11th Ship Control Systems Symp., Southampton, U.K., Apr. 14-18, 1997, vol. II, pp. 373-388.

[10] C. A. J. Tromp, J. M. Spoelstra, and J. K. Woud, "Torsional vibration analyzes as a tool for condition monitoring of piston engines by on-line cylinder torque estimation," presented at ICMES'96, The Institute of Marine Engineers, London, U.K., 1996, paper 15.

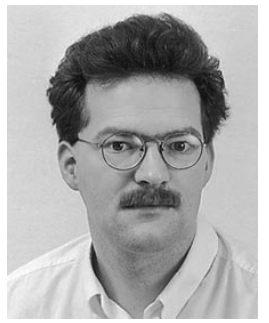

Hugo T. Grimmelius was born in Dinteloord, The Netherlands, in 1964. He received the B.Sc. degree in 1986 from Willem Barentsz Nautical College on the island of Terschelling, The Netherlands, and the M.Sc. degree in mechanical engineering in 1992 from Delft University of Technology, Delft, The Netherlands, where he is currently working towards the $\mathrm{Ph} . \mathrm{D}$. degree in the area of condition monitoring of refrigeration plants.

Following receipt of the B.Sc. degree, he sailed for a short period as a Second Engineer and completed military service. He then joined Delft University of Technology as a Research Assistant and also worked with a mechanical contractor. In 1997, he became an Assistant Professor of marine engineering and diesel engines in the Department of Marine Technology, Delft University of Technology. His research interests are condition monitoring, dynamic simulations, and system optimization. He is the author of several papers on condition monitoring and first principles modeling.

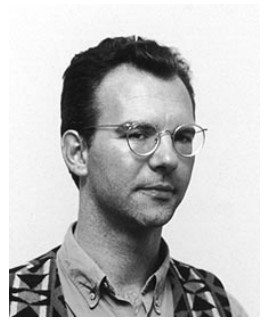

Peter P. Meiler was born in Rotterdam, The Netherlands, in 1962. He received the M.Sc. degree in electrical engineering from Delft University of Technology, Delft, The Netherlands, in 1987.

In 1987, he joined the High Performance Computing Group, TNO Physics and Electronics Laboratory, The Hague, The Netherlands, as a Scientist Since 1995, he has been with the Platform Command and Control Group. His research interests include signal processing, parallel processing, neural networks, artificial intelligence, and maritime command and control. He is the author of several papers on parallel processing, neural networks, signal processing, and maritime command and control.

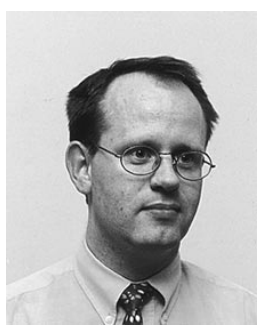

Hans L. M. M. Maas was born in Terneuzen, The Netherlands, in 1966. He received the M.Sc. degree in electrical engineering from Eindhoven University of Technology, Eindhoven, The Netherlands, in 1991.

In 1991, he joined the High Performance Computing Group, TNO Physics and Electronics Laboratory, The Hague, The Netherlands, as a Scientist Since 1995, he has been with the Platform Command and Control Group and, since 1997, he has been the Section Leader of the Combat Direction Systems Section. His research interests include signal processing, artificial intelligence, and maritime command and control. He is the author of several papers on signal processing and maritime command and control.

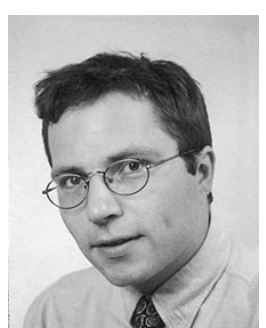

Bas Bonnier was born in Elburg, The Netherlands, in 1969. He received the M.Sc. degree in mechanical engineering from Delft University of Technology, Delft, The Netherlands, in 1995.

Following receipt of the M.Sc. degree, he joined the Design and Engineering Department, RDM Submarines, Rotterdam, The Netherlands, where he was involved with the development of a closedcycle diesel engine for air-independent propulsion of submarines. He is currently with MHI Equipment Europe, Almere, The Netherlands. His current research interest is automotive turbo chargers. He is author of several papers on diesel engine technology.

Mr. Bonnier is the recipient of an award from the Netherlands Society of Marine Engineers.

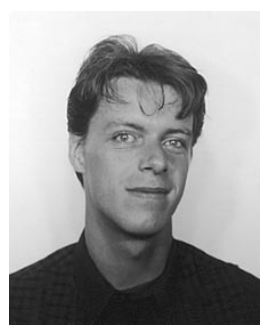

Jasper S. Grevink was born in Bussum, The Netherlands, in 1971. He received the M.Sc. degree in mechanical engineering from Delft University of Technology, Delft, The Netherlands, in 1996.

Following graduation, he joined Lips Propeller Works, Drunen, The Netherlands, where he is currently a Hydrodynamic Project Engineer. His current research interests are sensor fault detection, ship propulsion and maneuvering control, and propeller-induced pressure pulses.

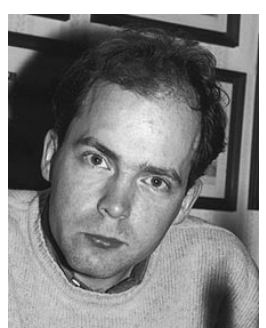

Robert F. van Kuilenburg was born in Berkel en Rodenrijs, The Netherlands, in 1972. He received the M.Sc. degree in mechanical engineering from Delft University of Technology, Delft, The Netherlands, in 1996.

Following graduation, he joined INNAS B.V., Breda, The Netherlands, to work on the free-piston engine and hydraulic transformers. His current research interest are combustion processes, emission control strategies, and hydraulic systems. 